\title{
3D Printed Silica Optical Fibre - a "Game Changer" Technology in Optical Fibre Manufacture
}

\author{
Yushi Chu ${ }^{1,2,3}$, Xinghu Fu ${ }^{1,4}$, Yanhua Luo ${ }^{1, *}$, John Canning ${ }^{2}$, Jianzhong Zhang ${ }^{3}$, Kevin Cook $^{2}$, and Gang- \\ Ding Peng ${ }^{1}$ \\ ${ }^{1}$ Photonics and Optical Communications, School of Electrical Engineering and Telecommunications, University of New South Wales, \\ Sydney, NSW 2052, Australia \\ ${ }^{2}$ interdisciplinary Photonics Laboratories, School of Electrical \& Data Engineering, University of Technology Sydney, NSW 2007 Australia \\ ${ }^{3}$ Key Laboratory of In-fiber Integrated Optics of Ministry of Education, College of Physics and Opto-Electric Engineering, Harbin \\ Engineering University, Harbin 150001, China \\ ${ }^{4}$ Key Laboratory for Special Fiber and Fiber Sensor of Hebei Province, School of Information Science and Engineering, Yanshan \\ University, Oinhuangdao, 066004, China \\ *Corresponding author: yanhua.luol@unsw.edu.au
}

\begin{abstract}
Based on 3D printing technology, multi-core doped silica optical fibre has been fabricated. The demonstration heralds a new fibre manufacturing milestone one that enables the design of fibres not previously feasible. (c) 2020 The Author(s)
\end{abstract}

\section{Introduction}

3D printing technology has significant advantages over traditional manufacturing methods. It increases design freedom for the allowance of complicated 3D geometries, faster prototyping, lower processing costs for small production volumes, more efficient material usage, the capability to manufacture parts from functionally graded materials and more. It has become a disruptive technology that is revolutionizing many areas from manufacturing, science and engineering to medicine and the arts. In optical fibre work, air-structured and step index polymer optical fibres have been produced using 3D printing [1,2]. Recently, a team in Germany reported the use of direct light projection to print small 3D glass objects with silica ink [3]. Such 3D silica lithography was proposed for future optical fibre manufacture [4]. On this basis, the world's first 3D printed single-mode and multimode silica fibres have recently been demonstrated [5]. Here, the design and fabrication of multi-core doped silica optical fibres by 3D printing using direct projection lithography (DLP) is evaluated.

\section{Fabrication of 3D Printed Silica Optical Fibres}

The fabrication process of 3D printing step index silica preform and fibre has been schemed in Fig. 1. AutoCAD software was used to design the preform. Given to the design freedom of 3D printing, arbitrary preform designs can be created that cannot be done by traditional fabrication means. Standard designs are also possible for structured optical fibres such as 2,3 and more ring-microstructured preforms and hollow core preform [6, 7]. For our work, 3D printing ink consisting of silica nanoparticles Aerosil OX50 (Evonik) and photocurable organic supports (monomer, cross-linker and, photoinitiators from Sigma Aldrich) was prepared. A 3D lithography commercial UV printer was used to build the cladding preform layer by layer. Subsequently, the preform core was doped with $\mathrm{GeO}_{2}$ and $\mathrm{TiO}_{2}$ and thermally consolidated in the oven. In this step, active dopants, like $\mathrm{Er}_{2} \mathrm{O}_{3}, \mathrm{Yb}_{2} \mathrm{O}_{3}, \mathrm{Bi}_{2} \mathrm{O}_{3}, \mathrm{ErCl}_{3}$, etc. can also be introduced to extend the functionality of the fibre. One example is the $\mathrm{Bi} / \mathrm{Er}$ co-doped optical fibre drawn from 3D printed silica preform, which gives out the typical emission at $\lambda=1430 \mathrm{~nm}$ for bismuth active centres and $1530 \mathrm{~nm}$ for the $\mathrm{Er}^{3+}$ ions [7, 8]. After preform consolidation, the organic carrier has been removed by thermal treatment of five stages in a furnace. This produces a sintered porous 3D printed silica preform. The sintered preform is inserted into a Heraeus F300 quartz tube, ready for the fibre drawing. The tube was sealed at the bottom end to allow pressure control during fibre drawing.

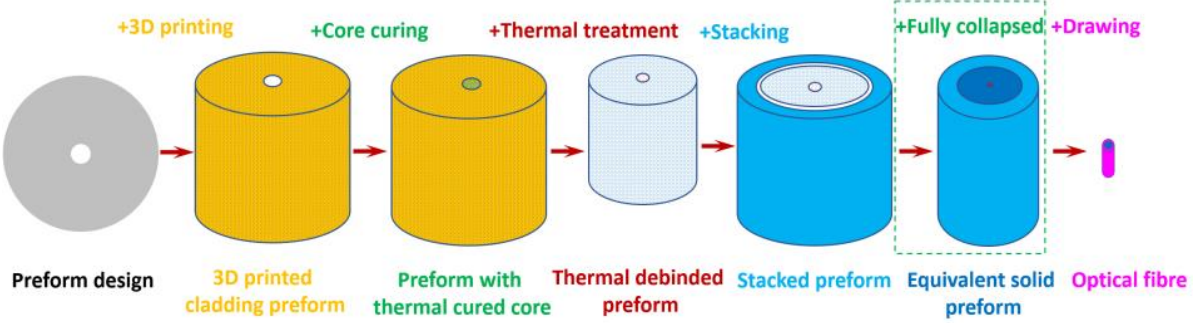

Fig. 1. Schematic flowchart of the fabrication of 3D printed optical fibre.

The 3D printed preform was mounted on the feeder of the fibre draw tower where the top end was connected with vacuum pump via a buffer tank. The pressure inside the tube was maintained $P \sim 50 \mathrm{mbar}$. To get rid of the humidity adsorbed by the materials, the drying process was performed at $T=820{ }^{\circ} \mathrm{C}$ at $P \sim 50$ mbar for several cycles. The temperature was increased to $T_{d}=1860{ }^{\circ} \mathrm{C}$ to melt the preform and start the 
drawing automatically. When the fibre is thinner to the requirement, the UV coating was applied to protect the bare fibre. By further adjusting drawing temperature $T_{d}$, preform feeding rate $v_{f}$, and fibre drawing rate $v_{d}$, a series of the 3D printed silica fibres have been drawn with a maximum drawing length $L \sim 2.3 \mathrm{~km}$.

Fig. 2 show the cross-section images of three 3D printed silica fibres drawn with different conditions. Seen from Fig. 2, the dimensions varied with the drawing conditions. All the fibre cores display a similar oval shape with good guidance property. By comparing the location along the drawn fibre, the 3D printed optical fibre is found to have very good consistency up to $L>500 \mathrm{~m}$. In addition, the transmission of $633 \mathrm{~nm}$ light in $2 \mathrm{~m}$ of $3 \mathrm{D}$ printed fibre is shown in Fig. 2(d). It indicates good quality waveguide structure of this 3D printed optical fibre.
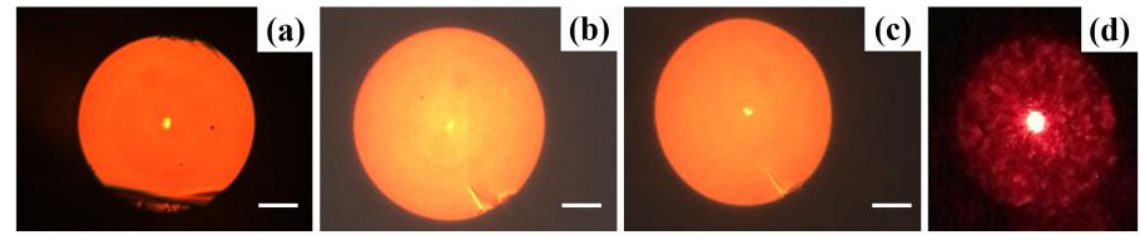

Fig. 2. The cross-section images of three 3D printed silica fibres drawn with different conditions $\left(L-\mathrm{m}, T_{d}-{ }^{\circ} \mathrm{C}, v_{f}-\right.$ $\mathrm{mm} / \mathrm{min}, v_{d}-\mathrm{mm} / \mathrm{min}$ ): (a) 0, 1854, 0.50,11.0; (b) 358, 1890, 0.61, 19.0; (c) 553, 1885, 0.55, 19.1. The scale is $20 \mu \mathrm{m}$. (d) The output pattern of $633 \mathrm{~nm}$ light transmitted through $2 \mathrm{~m}$ 3D printed fibre.

Preforms with two and seven cores were fabricated as well. The fibre cores have the dopants of bismuth and erbium. Through similar processes described in Fig. 1, 3D printed optical fibres with doped single, two and seven cores are fabricated, as demonstrated by the output pattern of $532 \mathrm{~nm}$ transmission in Fig. 3. There is shape variation arising from too high a drawing temperature.
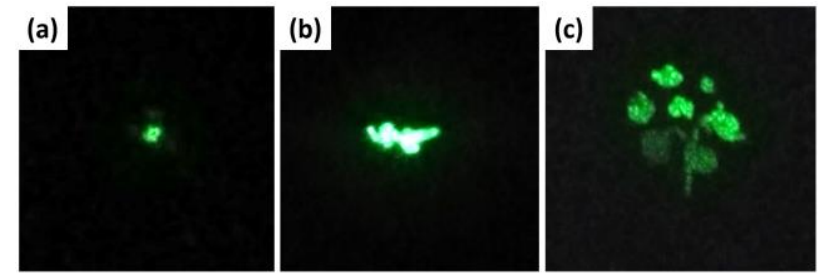

Fig. 3. The output pattern of 3D printed silica fibres with single (a), two (b) and seven (c) cores, transmitting $532 \mathrm{~nm}$ light.

\section{Conclusion}

The fabrication of silica preforms and fibres with doped single and multiple cores by 3D printing has been demonstrated. Given to the never-before-possible fibre design and flexible functionality by doping, 3D printing fibre fabrication will undoubtedly be a "game changer" in the history of optical fibres, greatly reducing the cost in terms of design, labor, fabrication and materials.

Acknowledgment: The authors thank the Australian Research Council (ARC) for funding the National Fiber Facility at UNSW (LE0883038, LE100100098). They thank the Air Force Office of Scientific Research (AFOSR) (FA2386-16-1-4031), the Asian Office of Aerospace R\&D (AOARD) and the High Energy Laser Joint Technology Office (HELJTO), United States, and the National Science Foundation, China (61775045).

\section{Reference}

[1] K. Cook et al., "Air-structured optical fiber drawn from a 3D-printed preform," Opt. Lett. 40, 3966-3969 (2015).

[2] K. Cook et al., "Step-index optical fiber drawn from 3D printed preforms," Opt. Lett. 41, 4554-4557 (2016).

[3] F. Kotz et al., "Three-dimensional printing of transparent fused silica glass," Nature 544, 337-342 (2017).

[4] G.-D. Peng et al., "3D Silica Lithography for future optical fiber fabrication", Handbook of Optical Fibers (Springer Singapore, Singapore, 2019), Vol. 1, 637.

[5] Y. Chu et al., "Silica optical fiber drawn from 3D printed preforms," Opt. Lett. 44, 5358-5361 (2019).

[6] Y. Chu et al., "3D printed optical fibre preforms from silica contained resin," in Sixth Workshop on Specialty Optical Fibers, J. Ballato and L. Dong, eds. (SPIE, Charleston, South Carolina, United States, 2019), p. 112061L.

[7] G.-D. Peng, "3D silica lithography for doped and structured optical fibres," in Sixth Workshop on Specialty Optical Fibers, J. Ballato and L. Dong, eds. (SPIE, Charleston, South Carolina, United States, 2019), p. 1120611.

[8] J. Canning, "3D Printing and Optical Waveguide Technologies”, 10 $0^{\text {th }}$ Int. Conf. Mater. for Adv. Technologies (ICMAT 2019), Singapore (23-28 June 2019) 